\title{
Microwave-assisted click chemistry: synthesis of mono and bis-1,2,3-triazole acyclonucleoside analogues of Acyclovir via copper(I)-catalyzed cycloaddition.
}

\author{
J. Krim, ${ }^{1}$ B. Sillahi, ${ }^{1}$ M. Taourirte, ${ }^{1 *}$ E. M. Rakib, ${ }^{2}$ and J. W. Engels ${ }^{3}$ \\ ${ }^{1}$ Laboratoire de chimie Bioorganique et Macromoléculaire; Faculté des Sciences et Techniques- \\ Guéliz, 40000, Marrakech, Maroc \\ ${ }^{2}$ Equipe d'Hétérochimie Organique, Faculté des Sciences et Techniques, Département de Chimie \\ et Environnement, B.P. 523, Béni-Mellal, Maroc \\ ${ }^{3}$ Institut für Organische Chemie und Chemische Biologie, J.W. Goethe Universität, Max-von- \\ Laue Str. 7, 60438 Frankfurt am Main, Germany \\ E-mail: taourirte@fstg-marrakech.ac.ma
}

\begin{abstract}
We report a short and efficient synthesis of 1,2,3-triazole and bis-1,2,3-triazoles acyclonucleoside analogues of Acycolovir. A series of novel 1,2,3-triazole acyclonucleosides linked to nucleobases were prepared via copper(I)-catalyzed 1,3-dipolar cycloaddition of N-9 propargylpurine, $\mathrm{N}$-1-propargylpyrimidines or N-1-propargylindazoles with the azido-pseudosugar under Microwave-assisted synthesis followed by treatment with $\mathrm{K}_{2} \mathrm{CO}_{3} / \mathrm{MeOH}$.
\end{abstract}

Keywords: 1,3-Dipolar cycloaddition, 1,2,3 triazole, 1,2,3-bis-triazoles, acyclovir, microwave

\section{Introduction}

Triazole heterocycles are found to be potent antimicrobial $1^{1}$, antiviral ${ }^{2}$, and anti-proliferative agents $^{3}$. The pharmaceutical importance of triazoles has prompted the design and synthesis of various triazolonucleosides (Figure 1). Ribavirin $\mathbf{1}$ was the first synthetic triazole nucleoside analogue found to show antiviral activity against many viruses ${ }^{4}$ and is still the only smallmolecular-weight drug available for treating viral infections caused by hepatitis $\mathrm{C}$ virus (HCV). In addition, 1,2,3 triazole TSAO [2',5'-bis- $O$-(tert-butyldimethylsilyl)- $\beta$-D-ribofuranosyl]-3'spiro-5"-(4"'-amino-1",2"'-oxathiole-2", 2-dioxide) 2 analogues exhibited an anti-HIV activity ${ }^{5}$. Moreover, recent studies have disclosed a series of 1,2,3-bis-triazoles as potent HIV-1 protease inhibitors for the inhibition of viral replication ${ }^{6}$. Since the discovery of acyclovir [9-((2hydroxyethoxy) methyl)guanine ACV (Zovirax)] 3 as the highly selective antiviral drug for the 
treatment of herpes simplex $(\mathrm{HSV})^{5}$ virus infections and Varicella-Zoster 1, several acyclonucleosides were synthesized and tested.

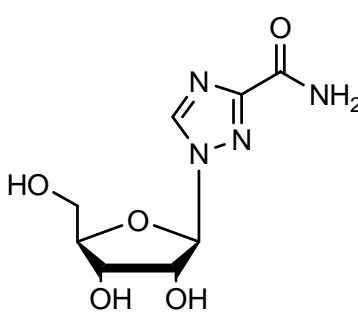

1 Ribavirin

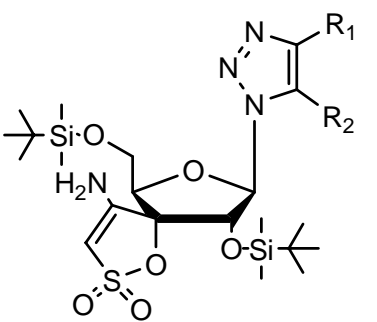

2 TSAO analogue

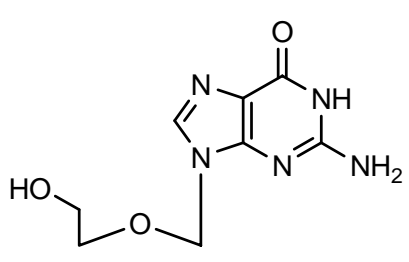

3 Acyclovir

Figure 1. Structure of Ribavirin 1, TSAO analogue 2 and Acyclovir 3.

In view of the biological importance of 1,2,3-triazoles and acyclovir, it was of considerable interest to develop a rapid, facile and practical protocol for the formation of compounds incorporating ring systems. In connection to our previous studies on the synthesis of acyclonucleosides, we report here a regioselective synthesis of 1,2,3-triazoles and bis-1,2,3triazole acyclonucleosides analogues of ACV in which the heterocyclic base is linked to a (2hydroxyethoxy)methyl via a methylene-1,2,3-triazole group. In this paper, we report an efficient approach for the one-pot synthesis of 1,4-disubstituted-1,2,3-triazoles analogues of ACV from propargylated bases and azido-pseudosugar under $\mathrm{CuI}$ catalysis.

\section{Results and Discussion}

The most widely used methods for the synthesis of triazoles is the Huisgen 1,3-dipolar cycloaddtion of alkynes with organic azides. This route to triazoles is often conducted at high temperature for a prolonged period of time, and usually leads to a mixture of 1,4-disubstituted and 1,5- disubstituted 1,2,3-triazoles. ${ }^{7}$ Copper-catalyzed click chemistry involving azides and terminal acetylenes has enabled practical and efficient preparation of 1,4-disubstituted-1,2,3triazoles, from a wide range of substrates with excellent selectivity. ${ }^{8-13}$ We decided to explore the feasibility of the "click"chemistry for the synthesis of novel 1,2,3-triazole acyclonucleosides with different heterocyclic bases connected via a flexible methylene linker. At first, several attempts were made in order to optimize the reaction conditions.

The synthetic approach to 1,4-disubstituted-1,2,3-triazoles analogues of ACV involved first the preparation of the known [(2-acetoxyethoxy)methyl]azide 6 from the [(2acetoxyethoxy)methyl]bromide 2 and sodium azide at $90-95^{\circ} \mathrm{C}$ for 4 h (Scheme 1$)^{7}$. 


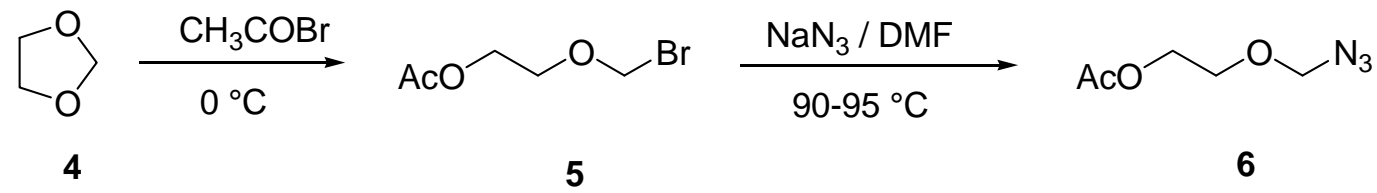

Scheme 1. Synthesis of the known azide $\mathbf{6}$ from bromide $\mathbf{5}$ and sodium azide.

The second step of the synthesis was the preparation the propargylated nucleobases. For this, uracil, thymine and iodouracil, were used as starting materials which were treated with propargylbromide in the presence of $\mathrm{K}_{2} \mathrm{CO}_{3}$. All reactions were carried out in DMF, as it is an excellent solvent for dissolving nucleobases ${ }^{7}$ (Scheme 2). The pyrimidine derivatives were exclusively alkylated at the N-1 position, 8a-c as confirmed by ${ }^{1} \mathrm{H}$ NMR spectra.

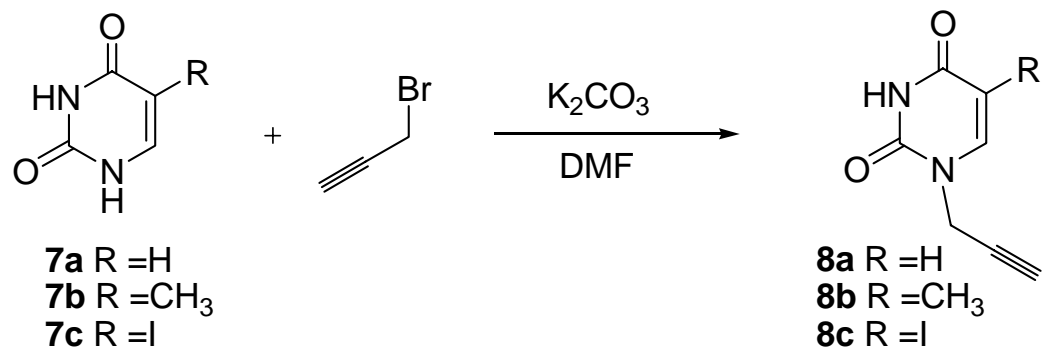

Scheme 2. N-1-alkylation of heterocyclic bases 7a-7c with propargylbromide.

Next, the terminal triple bonds of propargylated nucleobases were ligated to the azide residue of the pseudosugar using the copper(I)-catalyzed 1,3-dipolar cycloaddition in water-acetonitrile and $\mathrm{Et}_{3} \mathrm{~N}$ at room temperature leading to the 1,4-disubstituted regioisomer within $3 \mathrm{~h}$ (method A) ${ }^{14}$ ( 84 and $86 \%$ yields). In order to obtain these triazole acyclonucleosides in higher yields with shorter reaction times under mild reaction conditions, we turned our attention to microwave irradiation. The use of microwave-assisted organic synthesis has attracted considerable interest over the last two decades, leading often to remarkable decreases in reaction times, significant enhancements of yields, easier workups and better regioselectivity. ${ }^{15}$ 


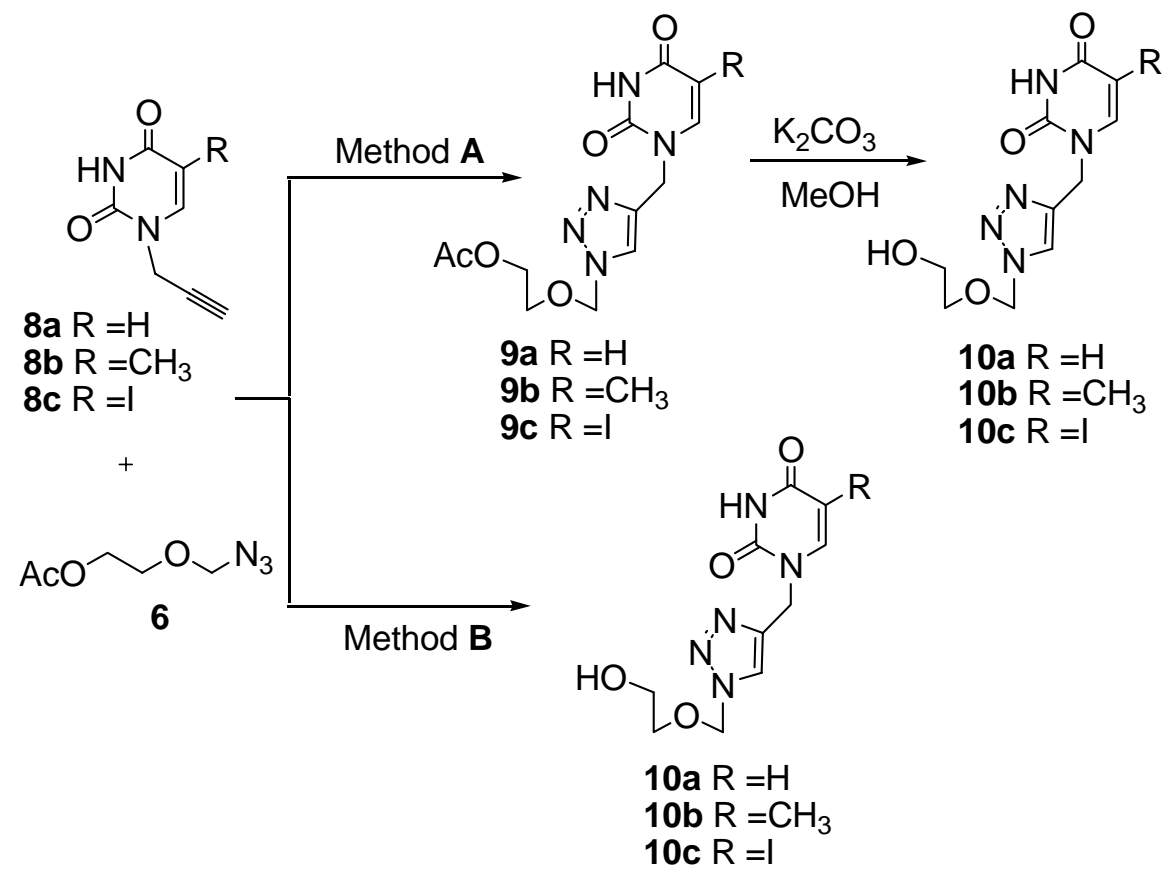

Scheme 3. Reagents and conditions: Method A: CuI, $\mathrm{Et}_{3} \mathrm{~N}, \mathrm{H}_{2} \mathrm{O} / \mathrm{CH}_{3} \mathrm{CN}, \mathrm{RT}$. Method B: 1) $\mathrm{MW}, \mathrm{CuI}, \mathrm{Et}_{3} \mathrm{~N}$; 2) $\mathrm{MeOH} / \mathrm{K}_{2} \mathrm{CO}_{3}$.

Herein, we report a rapid, facile and practical protocol for the formation of the triazole ring. The cycloaddition of propargylated nucleobases and azide carried out under microwave conditions with $\mathrm{CuI}$ as catalyst and without solvent lead to the desired products in a quantitative yield and reaction time of one minute (Scheme 3) (Method B). Consequently, Method B was selected as the best practical protocol for the synthesis of triazole acyclonucleosides, because it gave the best results.

Table 1. Synthesis of 1,4-disubstituted 1,2,3-triazole analogues of ACV catalysed by CuI under different reaction conditions

\begin{tabular}{|c|c|c|c|c|c|}
\hline \multirow[b]{2}{*}{ Compound } & \multirow{2}{*}{$\begin{array}{l}\text { Azide } \\
\text { equivalent }\end{array}$} & \multicolumn{2}{|l|}{ Method A } & \multicolumn{2}{|l|}{ MethodB } \\
\hline & & Yield (\%) & Reaction time & Yield (\%) & $\begin{array}{l}\text { Reaction } \\
\text { Time }\end{array}$ \\
\hline $10 a$ & 5 eq & 84 & $3 \mathrm{~h}$ & 92 & $1 \mathrm{~min}$ \\
\hline $10 b$ & $5 \mathrm{eq}$ & 86 & $3 \mathrm{~h}$ & 93 & $1 \mathrm{~min}$ \\
\hline 10c & $5 \mathrm{eq}$ & 85 & $3 \mathrm{~h}$ & 91 & $1 \mathrm{~min}$ \\
\hline
\end{tabular}

Method A: In water at room temperature.

Method B: under microwave 
To extend the general applicability of the microwave assisted click reaction, for the synthesis of triazole acyclonucleosides of purines and modified nucleobases.

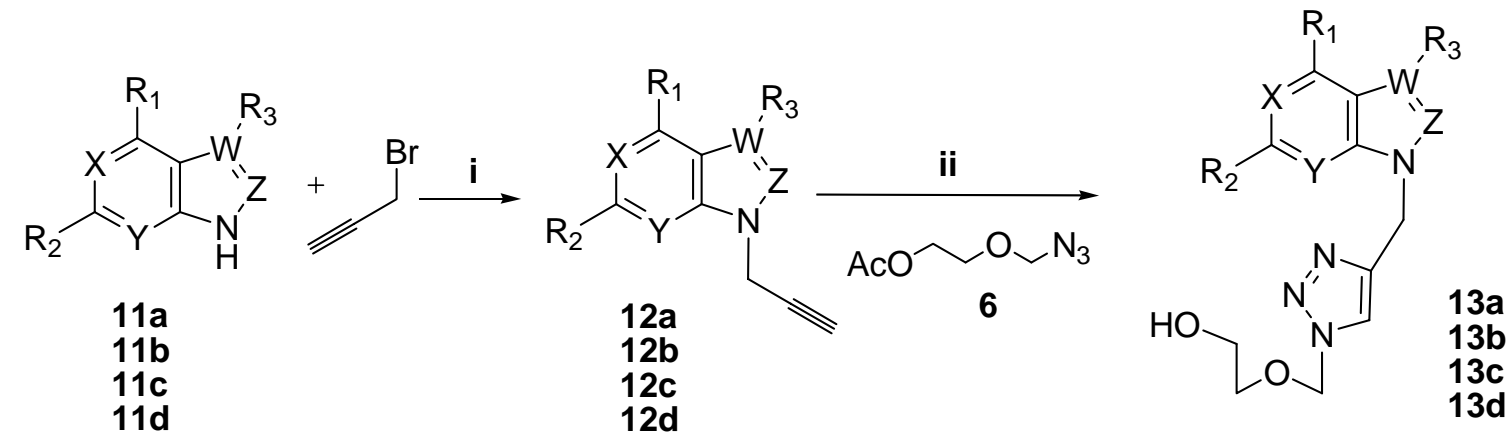

\begin{tabular}{llllllll}
\hline Compound & $\mathrm{X}$ & $\mathrm{Y}$ & $\mathrm{W}$ & $\mathrm{Z}$ & $\mathrm{R}_{1}$ & $\mathrm{R}_{2}$ & $\mathrm{R}_{3}$ \\
\hline 11a & $\mathrm{N}$ & $\mathrm{N}$ & $\mathrm{N}$ & $\mathrm{C}$ & $\mathrm{NH}_{2}$ & $\mathrm{H}$ & - \\
11b & $\mathrm{N}$ & $\mathrm{N}$ & $\mathrm{N}$ & $\mathrm{C}$ & $\mathrm{OH}$ & $\mathrm{NHAc}$ & - \\
11c & $\mathrm{C}$ & $\mathrm{C}$ & $\mathrm{C}$ & $\mathrm{N}$ & $\mathrm{H}$ & $\mathrm{NO}_{2}$ & $\mathrm{Cl}$ \\
11d & $\mathrm{C}$ & $\mathrm{C}$ & $\mathrm{C}$ & $\mathrm{N}$ & $\mathrm{H}$ & $\mathrm{NO}_{2}$ & $\mathrm{Br}$ \\
12a & $\mathrm{N}$ & $\mathrm{N}$ & $\mathrm{N}$ & $\mathrm{C}$ & $\mathrm{NH}_{2}$ & $\mathrm{H}$ & - \\
12b & $\mathrm{N}$ & $\mathrm{N}$ & $\mathrm{N}$ & $\mathrm{C}$ & $\mathrm{OH}$ & $\mathrm{NHAc}^{2}$ & - \\
12c & $\mathrm{C}$ & $\mathrm{C}$ & $\mathrm{C}$ & $\mathrm{N}$ & $\mathrm{H}$ & $\mathrm{NO}_{2}$ & $\mathrm{Cl}$ \\
12d & $\mathrm{C}$ & $\mathrm{C}$ & $\mathrm{C}$ & $\mathrm{N}$ & $\mathrm{H}$ & $\mathrm{NO}_{2}$ & $\mathrm{Br}$ \\
13a & $\mathrm{N}$ & $\mathrm{N}$ & $\mathrm{N}$ & $\mathrm{C}$ & $\mathrm{NH}_{2}$ & $\mathrm{H}$ & - \\
13b & $\mathrm{N}$ & $\mathrm{N}$ & $\mathrm{N}$ & $\mathrm{C}$ & $\mathrm{OH}$ & $\mathrm{NH}_{2}$ & - \\
13c & $\mathrm{C}$ & $\mathrm{C}$ & $\mathrm{C}$ & $\mathrm{N}$ & $\mathrm{H}$ & $\mathrm{NO}_{2}$ & $\mathrm{Cl}$ \\
13d & $\mathrm{C}$ & $\mathrm{C}$ & $\mathrm{C}$ & $\mathrm{N}$ & $\mathrm{H}$ & $\mathrm{NO}_{2}$ & $\mathrm{Br}$ \\
\hline
\end{tabular}

Scheme 4. Reagents and conditions: (i) $\mathrm{K}_{2} \mathrm{CO}_{3}$, DMF. (ii) 1) $\mathrm{CuI}$, $\mathrm{Et}{ }_{3} \mathrm{~N}$, $\mathrm{MW}$; 2) $\mathrm{MeOH} / \mathrm{K}_{2} \mathrm{CO}_{3}$.

$N$-9-propargyladenine, $N$-9-Propargyl- $N$-2-acetylguanine, $N$-1-propargyl-3-chloro and $N$-1propargyl-3-bromoindazole were prepared by using the same reaction conditions discussed above. Using the copper(I)-catalyzed 1,3-dipolar cycloaddition under click reaction conditions the desired products were obtained in a quantitative yield and reaction time of only one minute (Scheme 4).

With this promising procedure in hand, we extended the scope of substrates to include other alkinyle derivatives, as outlined in Scheme 5. Analogously to the preparation of N-1proparglated pyrimidine, the $\mathrm{N}-1, \mathrm{~N}-3$-bis-propargylated pyrimidines were prepared from starting material N-1-propargylated uracil, thymine and 5-iodouracil (Scheme 5), (Yields 80$88 \%$ ). The bis-propargylated pyrimidines were converted into the bis-triazoles acyclonucleosides using the same reaction conditions in quantitative yield. 
<smiles>[R]c1cn(CC#C)c(=O)[nH]c1=O</smiles>

$8 a \mathrm{R}=\mathrm{H}$

8b $\mathrm{R}=\mathrm{CH}_{3}$

$8 c R=1$

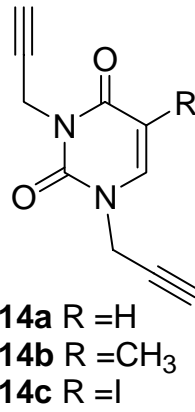

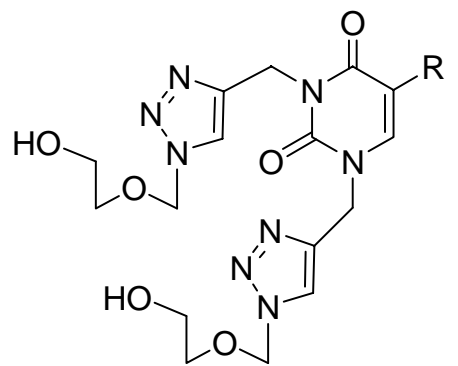

$15 \mathrm{a} \mathrm{R}=\mathrm{H}$ 15b $\mathrm{R}=\mathrm{CH}_{3}$ $15 c \mathrm{R}=\mathrm{I}$

Scheme 5. Reagents and conditions: (i) $\mathrm{K}_{2} \mathrm{CO}_{3}$, DMF. (ii) 1) $\mathrm{CuI}, \mathrm{Et}_{3} \mathrm{~N}, \mathrm{MW}$; 2) $\mathrm{MeOH} / \mathrm{K}_{2} \mathrm{CO}_{3}$

Using the copper(I)-catalyzed 1,3-dipolar cycloaddition under click reaction conditions the bis-propargyl-5-iodouracil 14c unexpectedly leads to bis-triazoluracil 15a in a quantitative yield and reaction time of one minute. This structure was established on the basis of ${ }^{1} \mathrm{H},{ }^{13} \mathrm{C}$ NMR spectra, mass spectrum and comparison with the spectroscopic data of 15a. This transformation can best be explained by a deiodination of N-1, N-3-bis-alkylated 5-iodouracil in the presence of CuI.

The structure of all compounds was confirmed on the basis of ${ }^{1} \mathrm{H},{ }^{13} \mathrm{C}$ NMR spectra and mass spectra. The formation of 1,4-disubstituted triazoles was unequivocally established through the characteristic chemical shift value of the triazolyl proton $(5-\mathrm{CH})$ at $\delta=8.07-8.54 \mathrm{ppm}$. The triazole ring formation can also be identified from the $13 \mathrm{C}$-spectra with the new signals of the olefinic C-atoms of the 1,2,3-triazole moiety at $(\delta(\mathrm{C} 5)=122.5-125 \mathrm{ppm})$ and $(\delta(\mathrm{C} 4)=140-144$ ppm).

Table 2. Synthesis of 1,4-disubstituted 1,2,3-triazoles analogue of ACV catalysed by CuI under microwave irradiation (Method B).

\begin{tabular}{llll}
\hline Compound & $\begin{array}{l}\text { Azide } \\
\text { equivalent }\end{array}$ & $\begin{array}{l}\text { Reaction } \\
\text { time }\end{array}$ & Yield (\%) \\
\hline 13a & 5 eq & $1 \mathrm{~min}$ & 92 \\
13b & 5 eq & $1 \mathrm{~min}$ & 90 \\
13c & $5 \mathrm{eq}$ & $1 \mathrm{~min}$ & 94 \\
13d & $5 \mathrm{eq}$ & $1 \mathrm{~min}$ & 93 \\
15a & $5 \mathrm{eq}$ & $1 \mathrm{~min}$ & 91 \\
15b & $5 \mathrm{eq}$ & $1 \mathrm{~min}$ & 90 \\
\hline
\end{tabular}




\section{Conclusions}

In summary, we report a short and efficient synthesis of 1,2,3-triazole and bis-1,2,3-triazoles acyclonucleoside analogues of ACV. We accomplished a straightforward convenient methodology for the synthesis of novel 1,2,3-triazole nucleosides using the $\mathrm{Cu}(\mathrm{I})$-catalyzed alkyne-azide cycloaddition reaction. These products are under investigation for their biological activities.

\section{Experimental Section}

General. Melting points were determined in open capillary tubes and are uncorrected. NMR spectra were recorded at $300 \mathrm{MHz}\left({ }^{1} \mathrm{H},{ }^{13} \mathrm{C}\right)$ Bruker in $\left(\right.$ DMSO- $\left._{6}, \mathrm{CDCl}_{3}\right)$ using TMS as an internal reference. All chemical shifts $(\delta)$ are expressed in parts per million and coupling constant $(J)$ are given in Hertz; T (1,2,3-triazole) and B (heterocyclic base). Mass spectra were obtained by using ESI/MS and $\left(\mathrm{FAB}^{+}\right)$. Reactions were performed in a domestic microwave oven Model AVM510/WP/WH. DMF and MeCN were distilled prior to use and stored over molecular sieves 4A. Precoated Merck Silica Gel 60F-254 plates were used for thin layer chromatography (TLC) and the spots were detected under UV light $(254 \mathrm{~nm})$. Column chromatography (CLC) was performed using silica gel (0.063-0.2 mm) Fluka. All reagents used were purchased from Aldrich.

[(2-Acetoxyethoxy)methyl]bromide ${ }^{7}$. To 106 mmol (13 g) of distilled acetyl bromide, was added $100 \mathrm{mmol}$ of 1,3-dioxolane drop wise while agitating and cooling by a bath of ice. The reaction is fast and exothermic. After the addition, the reaction is agitated further during $30 \mathrm{~min}$ at room temperature. Afterwards the reaction mixture was distilled under reduced pressure. ${ }^{1} \mathrm{H}$ NMR, $\mathrm{CDCl}_{3}, \delta 2.1$ (s, 3H, $\left.\mathrm{CH}_{3} \mathrm{COO}\right), 3.88\left(\mathrm{t}, 2 \mathrm{H}, \mathrm{OCH}_{2}\right), 4.27\left(\mathrm{t}, 2 \mathrm{H}, \mathrm{CH}_{2} \mathrm{O}\right), 5.72(\mathrm{~s}, 2 \mathrm{H}$, $\mathrm{OCH}_{2} \mathrm{Br}$ ).

[(2-acetoxyethoxy)methyl]azide ${ }^{7}$ 6. To a solution of $10 \mathrm{mmol}(2 \mathrm{~g})$ of [(2-acetoxyethoxy) methyl]bromide in $60 \mathrm{ml}$ of anhydrous DMF is added $15 \mathrm{mmol}(0,9 \mathrm{~g})$ of sodium azide $\left(\mathrm{NaN}_{3}\right)$. The mixture was brought up to a temperature of $90-95^{\circ} \mathrm{C}$ during $4 \mathrm{~h}$. After cooling, the solution was extracted with ether $(2 \times 50 \mathrm{ml})$ then washed with brine, and dried $\left(\mathrm{MgSO}_{4}\right)$. After removal of the solvent under reduced pressure, the residual oil was purified on a silica gel column with hexane (95\%). ${ }^{1} \mathrm{H} \mathrm{NMR}, \mathrm{CDCl}_{3}, \delta 2.1$ (s, 3H, $\left.\mathrm{CH}_{3} \mathrm{COO}\right) ; 3.8\left(\mathrm{t}, 2 \mathrm{H}, \mathrm{OCH}_{2}\right) ; 4.2\left(\mathrm{t}, 2 \mathrm{H}, \mathrm{CH}_{2} \mathrm{O}\right)$; $4.65\left(\mathrm{~s}, 2 \mathrm{H}, \mathrm{OCH}_{2} \mathrm{~N}_{3}\right)$.

\section{General procedure for the synthesis of the monopropargyl heterocyclic bases ${ }^{7}$}

The mixture of $1 \mathrm{mmol}$ of the heterocyclic base (thymine, uracil, iodouracil, adenine, N2acetylguanine, 3-bromo-6-nitroindazole or 3-chloro-6- nitroindazole), 0,5 mmol of $\mathrm{K}_{2} \mathrm{CO}_{3}$ and $1 \mathrm{mmol}$ of propargylbromide in $20 \mathrm{ml}$ of anhydrous DMF was stirred at room temperature 
during 24h. After removal of the solvent under reduced pressure the residue obtained was purified on silica gel column $\mathrm{CH}_{2} \mathrm{Cl}_{2}$ and $\mathrm{MeOH}(99 / 1)$.

$N$-1-Propargyluracil 8a. ( 54 \%), Solid, mp $154-166^{\circ} \mathrm{C},{ }^{1} \mathrm{H}$ NMR, DMSO-d 6 , $\delta 3.43(\mathrm{t}, 1 \mathrm{H}, \mathrm{CH}$, $J=2.3 \mathrm{~Hz}) ; 4.57\left(\mathrm{~d}, 2 \mathrm{H}, \mathrm{CH}_{2} \mathrm{~N}, J=2.3 \mathrm{~Hz}\right) ; 5.7(\mathrm{~d}, 1 \mathrm{H}, \mathrm{H}-5, J=7.8 \mathrm{~Hz}) ; 7.75(\mathrm{~d}, 1 \mathrm{H}, \mathrm{H}-6, J=7.8$ $\mathrm{Hz}) ; 11.44(\mathrm{~s}, 1 \mathrm{H}, \mathrm{NH}) .{ }^{13} \mathrm{C}$ NMR, DMSO-d 6 : 36.60; 75.79; 78.42; 101.67; 144.42; 150.35; 163.50. FAB-MS, $\mathrm{C}_{7} \mathrm{H}_{6} \mathrm{~N}_{2} \mathrm{O}_{2} \mathrm{~m} / \mathrm{z} 151(\mathrm{M}+\mathrm{H})^{+}$.

$N$-1-Propargylthymine 8b. (56\%), Solid, mp $154-156{ }^{\circ} \mathrm{C},{ }^{1} \mathrm{H}$ NMR, DMSO-d 6 , $\delta 1.75$ (s, 3H, $\left.\mathrm{CH}_{3}\right) ; 3,37$ (t, $\left.1 \mathrm{H}, \mathrm{CH}, J=2.2 \mathrm{~Hz}\right) ; 4.46\left(\mathrm{~d}, 2 \mathrm{H}, \mathrm{CH}_{2} \mathrm{~N} J=7,8 \mathrm{~Hz}\right) ; 7.55(\mathrm{~s}, 1 \mathrm{H}, \mathrm{H}-6) ; 11.35$ (s, $1 \mathrm{H}, \mathrm{NH}) .{ }^{13} \mathrm{C}$ NMR, DMSO-d 6 : $11.87 ; 36.30 ; 75.58 ; 78.61 ; 109.38 ; 140.04 ; 150.33 ; 164.09$. FAB-MS, $\mathrm{C}_{8} \mathrm{H}_{8} \mathrm{~N}_{2} \mathrm{O}_{2} \mathrm{~m} / \mathrm{z} 165(\mathrm{M}+\mathrm{H})^{+}$.

$N$-1-Propargyl-5-iodouracil 8c. (62\%), Solid, mp180-182 ${ }^{\circ} \mathrm{C},{ }^{1} \mathrm{H}$ NMR, DMSO-d ${ }_{6}, \delta 3.42(\mathrm{t}$, $1 \mathrm{H}, \mathrm{CH}, J=2.6 \mathrm{~Hz}) ; 4.58\left(\mathrm{~d}, 2 \mathrm{H}, \mathrm{CH}_{2} \mathrm{~N}, J=2.6 \mathrm{~Hz}\right) ; 8.28(\mathrm{~d}, 1 \mathrm{H}, \mathrm{H}-6) ; 12.02(\mathrm{~s}, 1 \mathrm{H}, \mathrm{NH}) .{ }^{13} \mathrm{C}$ NMR, DMSO-d $\mathrm{d}_{6}: 36.94 ; 68.85 ; 75.98 ; 78.31 ; 148.69 ; 150.05 ; 160.87$. FAB-MS, $\mathrm{C}_{7} \mathrm{H}_{5} \mathrm{IN}_{2} \mathrm{O}_{2} \mathrm{~m} / \mathrm{z}$ $277(\mathrm{M}+\mathrm{H})^{+}$.

$N$-9-Propargyladenine 12a. (80\%) Solid, mp $213-214{ }^{\circ} \mathrm{C},{ }^{1} \mathrm{H}$ NMR, DMSO- $\mathrm{d}_{6}, \delta: 3.56(\mathrm{t}, 1 \mathrm{H}$, $\mathrm{CH}) ; 5.03\left(\mathrm{~d}, 2 \mathrm{H}, \mathrm{CH}_{2}-\mathrm{N}\right) ; 7.3\left(\mathrm{~s}, 2 \mathrm{H}, \mathrm{NH}_{2}\right) ; 8.18(2 \mathrm{~s}, 2 \mathrm{H}, \mathrm{H}-2$ and $\mathrm{H}-8) .{ }^{13} \mathrm{C}$ NMR, DMSO-d 6 : $32.23 ; 75.81 ; 78.29 ; 118.51 ; 140.07 ; 149.08 ; 152.69 ; 155.99$. FAB-MS, $\mathrm{C}_{8} \mathrm{H}_{7} \mathrm{~N}_{5} \mathrm{~m} / \mathrm{z} \quad 174$ $(\mathrm{M}+\mathrm{H})^{+}$.

N-9-Propargyl-N-2-acetylguanine 12b. (40\%), Solid, mp 285-286 ${ }^{\circ} \mathrm{C},{ }^{1} \mathrm{H}$ NMR, DMSO- $\mathrm{d}_{6}, \delta$ $2.2\left(\mathrm{~s}, 3 \mathrm{H}, \mathrm{CH}_{3} \mathrm{CON}\right) ; 3.55$ (t, 1H, CH); $4.95\left(\mathrm{~d}, 2 \mathrm{H}, \mathrm{CH}_{2}-\mathrm{N}\right) ; 7.35\left(\mathrm{bs}, 2 \mathrm{H}, \mathrm{NH}_{2}\right) ; 8.08(\mathrm{~s}, 1 \mathrm{H}, \mathrm{H}-$ 8). ${ }^{13} \mathrm{C}$ NMR, DMSO- $\mathrm{d}_{6}: 25.36 ; 32.90 ; 72.85 ; 78.30 ; 117.51 ; 143.60 ; 152.43 ; 153.30 .158 .58$; 170.63. FAB-MS, $\mathrm{C}_{10} \mathrm{H}_{9} \mathrm{~N}_{5} \mathrm{O}_{2} \mathrm{~m} / \mathrm{z} 233(\mathrm{M}+\mathrm{H})^{+}$.

$\mathrm{N}$-1-Propargyl(3-bromo-6-nitroindazole) 12c. (91\%) Solid, mp $128{ }^{\circ} \mathrm{C},{ }^{1} \mathrm{H}$ NMR, DMSO-d 6 , $\delta 3.50(\mathrm{t}, 1 \mathrm{H}, \mathrm{CH}) ; 5.55\left(\mathrm{~d}, 2 \mathrm{H}, \mathrm{CH}_{2}-\mathrm{N}\right) ; 7.86-8.07(\mathrm{~m}, 2 \mathrm{H}, \mathrm{H} 5-, \mathrm{H}-4) ; 8.90(\mathrm{~d}, 1 \mathrm{H}, \mathrm{H}-7),{ }^{13} \mathrm{C} \mathrm{NMR}$, DMSO-d 6 : 40.91; 77.23; 78.42; 108.19; 117.13; 121.44; 121.96; 126.79; 139.47; 147.82.

$\mathrm{N}$-1-Propargyl(3-chloro-6-nitroindazole) 12d. (90\%) Solid, mp $121{ }^{\circ} \mathrm{C},{ }^{1} \mathrm{H}$ NMR, DMSO-d 6 3.51( t, $1 \mathrm{H}, \mathrm{CH}) ; 5.554\left(\mathrm{~d}, 2 \mathrm{H}, \mathrm{CH}_{2}-\mathrm{N}\right) ; 7.95-8.05(\mathrm{~m}, 2 \mathrm{H}, \mathrm{H}-5, \mathrm{H}-4) ; 8.89(\mathrm{~d}, 1 \mathrm{H}, \mathrm{H}-7),{ }^{13} \mathrm{C} \mathrm{NMR}$, DMSO-d 6 : $41.55 ; 77.89 ; 79.01 ; 108.99 ; 117.74 ; 122.02 ; 124.68 ; 133.98 ; 140.47 ; 148.48$.

\section{General procedure for the synthesis of the N-1, N-3-bis-propargylpyrimidines}

The mixture of $1 \mathrm{mmol}$ of the heterocyclic base ( $\mathrm{N}$-1-propargyluracil, $\mathrm{N}$-1-propargylthymine, $\mathrm{N}$ 1-propargyl-5-iodouracil), $0,5 \mathrm{mmol}$ of $\mathrm{K}_{2} \mathrm{CO}_{3}$ and $1.1 \mathrm{mmol}$ of propargylbromide in $20 \mathrm{ml}$ of anhydrous DMF was stirred at room temperature during $15 \mathrm{~h}$. After removal of the solvent under reduced pressure and purification on silica gel column chromatography, we obtained the desired pure product.

$N$-1, $N$-3-Dipropargyluracil 14a. (80\%), Solid, mp $105^{\circ} \mathrm{C},{ }^{1} \mathrm{H}$ NMR, DMSO-d $\mathrm{d}_{6}, \delta 3.10(\mathrm{t}, 1 \mathrm{H}$, $\mathrm{CH}) ; 3.44(\mathrm{t}, 1 \mathrm{H}, \mathrm{CH}) ; 4.52\left(\mathrm{~d}, 2 \mathrm{H}, \mathrm{CH}_{2} \mathrm{~N}\right) ; 4.59\left(\mathrm{~d}, 2 \mathrm{H}, \mathrm{CH}_{2} \mathrm{~N}\right) ; 5.81(\mathrm{~d}, 1 \mathrm{H}, \mathrm{H}-5, J=8.1 \mathrm{~Hz})$; $7,79$ ( d,1H, H-6, $J=7.8 \mathrm{~Hz}){ }^{13} \mathrm{C}$ NMR, DMSO-d $6: 31,06 ; 31,90 ; 74,24 ; 77.42 ; 79.37 ; 80.14$; $101.99 ; 144.76 ; 151.11 ; 162.51$. 
$\mathrm{N}$-1,N-3-Dipropargylthymine 14b. (84\%), Solid, mp $101{ }^{\circ} \mathrm{C},{ }^{1} \mathrm{H}$ NMR, DMSO- $\mathrm{d}_{6}, \delta 1.83$ (s, $\left.3 \mathrm{H}, \mathrm{CH}_{3}\right) ; 3.08(\mathrm{t}, 1 \mathrm{H}, \mathrm{CH}) ; 3.39(\mathrm{t}, 1 \mathrm{H}, \mathrm{CH}) ; 4.55\left(\mathrm{~m}, 4 \mathrm{H}, 2 \mathrm{x}\left(\mathrm{CH}_{2} \mathrm{~N}\right)\right) ; 7.67(\mathrm{~s}, 1 \mathrm{H}, \mathrm{H}-6),{ }^{13} \mathrm{C}$ NMR, DMSO-d 6 : $13.03 ; 30.68 ; 38.17 ; 73.54 ; 76.56 ; 78.90 ; 79.60 ; 109.19 ; 139.82 ; 150.31$; 162.64 .

$N$-1,N-3-Dipropargyliodouracil 14c. (88\%), Solid, mp $123-126{ }^{\circ} \mathrm{C},{ }^{1} \mathrm{H}$ NMR, DMSO- $\mathrm{d}_{6}, \delta$ $3.14(\mathrm{t}, 1 \mathrm{H}, \mathrm{CH}) ; 3.45(\mathrm{t}, 1 \mathrm{H}, \mathrm{CH}) ; 4.55\left(\mathrm{~m}, 4 \mathrm{H}, 2 \mathrm{x}\left(\mathrm{CH}_{2} \mathrm{~N}\right)\right) ; 8,33(\mathrm{~d}, 1 \mathrm{H}, \mathrm{H}-6, J=7.8 \mathrm{~Hz}),{ }^{13} \mathrm{C}$ NMR, DMSO-d $\mathrm{d}_{6}: 31.46 ; 38.19 ; 67.50 ; 73.43 ; 76.31 ; 78.06 ; 78.58 ; 147.77 ; 149.47 ; 158.99$.

\section{General procedure for the synthesis of the triazole acyclonucleoside derivatives}

The synthesis of the 1,2,3-triazole derivatives was carried out by two methods:

Method A. To a solution of $5 \mathrm{mmol}$ of the alkylazide in $10 \mathrm{ml}$ of water/acetonitrile (v/v) was added $1 \mathrm{mmol}$ of N-propargylbase, $1 \mathrm{mmol} \mathrm{Et}_{3} \mathrm{~N}$ and $0,1 \mathrm{mmol}$ of $\mathrm{CuI}$ for $3 \mathrm{~h}$ at room temperature. The reaction mixture was diluted with $25 \mathrm{~mL}$ of water, cooled in ice, and extracted with $\mathrm{CH}_{2} \mathrm{Cl}_{2}$ $(3 \times 15 \mathrm{ml})$. The combined organic extracts were washed with brine, and dried $\left(\mathrm{MgSO}_{4}\right)$. After removal of the solvent under reduced pressure, the acetyl groups of the protected nucleosides were then cleaved using $2 \mathrm{mmol}$ of $\mathrm{K}_{2} \mathrm{CO}_{3}$ in $10 \mathrm{ml}$ of methanol, and the reaction mixture was stirred for $3 \mathrm{~h}$ at room temperature. When TLC analysis showed no more starting material, solvent was removed by rotary evaporation, and the residue was purified on silica gel with dichloromethane and methanol to give the desired compound.

Method B. the mixture of alkylazide $(5 \mathrm{mmol}), \mathrm{Et}_{3} \mathrm{~N}(1 \mathrm{mmol})$, N-propargylbase $(1 \mathrm{mmol})$ and $0,1 \mathrm{mmol}$ of $\mathrm{CuI}$ were irradiated in the microwave oven at power level $(300 \mathrm{~W})$ for $1 \mathrm{~min}$ without solvent. $\mathrm{K}_{2} \mathrm{CO}_{3}(2 \mathrm{mmol})$ in $10 \mathrm{ml}$ of methanol were added directly to reaction mixture. The mixture was stirred for additional $3 \mathrm{~h}$ at room temperature. When TLC analysis showed no starting material, solvent was removed under reduced pressure, and the residue was purified on silica gel with dichloromethane and methanol.

1-[[1-[(2-Hydroxyethoxy)methyl]-1,2,3-triazol-4-yl]methyl]uracil 10a. Solid, mp 154 $156{ }^{\circ} \mathrm{C},{ }^{1} \mathrm{H}$ NMR, DMSO-d 6 , $\delta 3.47$ ( m, 4H, $\left.\mathrm{CH}_{2} \mathrm{CH}_{2}\right), 4.67$ ( s, $\left.1 \mathrm{H}, \mathrm{OH}\right), 4.95$ (s, 2H, T- $\mathrm{CH}_{2}-$ B), 5.7(s, 2H, OCH $-\mathrm{T}) ; 5.72(\mathrm{~d}, 1 \mathrm{H}, \mathrm{H}-5, J=7,4 \mathrm{~Hz}) ; 7.75(\mathrm{~d}, 1 \mathrm{H}, \mathrm{H}-6, J=7,5 \mathrm{~Hz}), 8.2(\mathrm{~s}$, $1 \mathrm{H}, \mathrm{H}-5$ (triazole)), 11.3 (s, $1 \mathrm{H}, \mathrm{NH}) .{ }^{13} \mathrm{C}$ NMR, DMSO-d 6 : 42.31; 59.70; 70.95; 78.20; 101.25; $123.97 ; 142.76 ; 145.48 ; 150.71 ; 163.69$. ESI-MS, $\mathrm{C}_{10} \mathrm{H}_{13} \mathrm{~N}_{5} \mathrm{O}_{4} \mathrm{~m} / \mathrm{z}=267$.

1-[[1-[(2-Hydroxyethoxy)methyl]-1,2,3-triazol-4-yl]methyl]thymine 10b. Solid, mp 118 $120{ }^{\circ} \mathrm{C},{ }^{1} \mathrm{H}$ NMR, DMSO-d 6 , $\delta 1.7$ (s, 3H, $\left.\mathrm{CH}_{3}\right), 3.46\left(\mathrm{~m}, 4 \mathrm{H}, \mathrm{CH}_{2} \mathrm{CH}_{2}\right), 4.57(\mathrm{~s}, 1 \mathrm{H}, \mathrm{OH})$, $4.91\left(\mathrm{~s}, 2 \mathrm{H}, \mathrm{T}-\mathrm{CH}_{2}-\mathrm{B}\right), 5.69\left(\mathrm{~s}, 2 \mathrm{H}, \mathrm{OCH}_{2} \mathrm{~N}\right) ; 7.62(\mathrm{~s}, 1 \mathrm{H}, \mathrm{H}-6), 8.21(\mathrm{~s}, 1 \mathrm{H}, \mathrm{H} 5$ triazole), 11.31 $(\mathrm{s}, 1 \mathrm{H}, \mathrm{NH}){ }^{13} \mathrm{C}$ NMR, DMSO-d 6 : 12.50; 42.74; 63.16; 67.73; 78.50; 109.47; 124.61; 141.72; 143.16; 151.28; 164.84, ESI-MS; $\mathrm{C}_{11} \mathrm{H}_{15} \mathrm{~N}_{5} \mathrm{O}_{4} \mathrm{~m} / \mathrm{z}=281$.

1-[[1-[(2-Hydroxyethoxy)methyl]-1,2,3-triazol-4-yl]methyl]iodouracil 10c. Solid, mp $155^{\circ} \mathrm{C}$, ${ }^{1} \mathrm{H}$ NMR, DMSO-d ${ }_{6}, \delta 3.71\left(\mathrm{~m}, 4 \mathrm{H}, \mathrm{CH}_{2} \mathrm{CH}_{2}\right) ; 4.92(\mathrm{~m}, 1 \mathrm{H}, \mathrm{OH}) ; 5.18\left(\mathrm{~s}, 2 \mathrm{H}, \mathrm{T}_{-} \mathrm{CH}_{2}-\mathrm{B}\right)$, 5.91(s, 2H, $\left.\mathrm{OCH}_{2}-\mathrm{T}\right) ; 8.44$ (s, $1 \mathrm{H}, \mathrm{H}-5$ (triazole)); 8.53 (s, 1H, H-6); $11.3(\mathrm{~s}, 1 \mathrm{H}, \mathrm{NH}) .{ }^{13} \mathrm{C} \mathrm{NMR}$, DMSO-d 6 : 43.20; 60.32; 69.09; 71.57; 78.82; 124.61; 143.24; 150.31; 151.01; 161.64. ESI-MS, $\mathrm{C}_{10} \mathrm{H}_{12} \mathrm{~N}_{5} \mathrm{O}_{4} \mathrm{I} \mathrm{m} / \mathrm{z}=393.7$. 
9-[[1-[(2-Hydroxyethoxy)methyl]-1,2,3-triazol-4-yl]methyl]adenine 13a. Solid, mp 209 $212{ }^{\circ} \mathrm{C},{ }^{1} \mathrm{H}$ NMR, DMSO-d 6 , $\delta: 3.4\left(\mathrm{~m}, 4 \mathrm{H}, \mathrm{CH}_{2} \mathrm{CH}_{2}\right), 4.65(\mathrm{~s}, 1 \mathrm{H}, \mathrm{OH}), 5.45\left(\mathrm{~s}, 2 \mathrm{H}, \mathrm{T}_{-} \mathrm{CH}_{2}-\right.$ B), $5.7\left(\mathrm{~s}, 2 \mathrm{H}, \mathrm{OCH}_{2} \mathrm{~N}\right) ; 7.22\left(\mathrm{~s}, 2 \mathrm{H}, \mathrm{NH}_{2}\right), 8.05(\mathrm{~s}, 1 \mathrm{H}, \mathrm{H}-5$ (triazole)), 8.2 and $8.25(\mathrm{~s}, 2 \mathrm{H}, \mathrm{H}-$ 2 and $\mathrm{H}-8)$. FAB-MS, $\mathrm{C}_{11} \mathrm{H}_{14} \mathrm{~N}_{8} \mathrm{O}_{2} \mathrm{~m} / \mathrm{z} 291(\mathrm{M}+\mathrm{H})^{+}$.

9-[[1-[(2-Hydroxyethoxy)methyl]-1,2,3-triazol-4-yl]methyl]guanine 13c. Solid, mp 209 $212{ }^{\circ} \mathrm{C},{ }^{1} \mathrm{H}$ NMR, DMSO-d 6 , $\delta 3.5$ (m, 4, $\mathrm{CH}_{2} \mathrm{CH}_{2}$ ); 4.65 (sl, 1, OH); 5.55 (s, 2, T- $\mathrm{CH}_{2}-\mathrm{B}$ ); 5.7 (s, 2, $\left.\mathrm{OCH}_{2} \mathrm{~N}\right) ; 6.3$ (bs, 2, $\mathrm{NH}_{2}$ ); 8.0 (s, 1H, H-5(triazole)); 8.2 (s, 1, H-8); FAB-MS, $\mathrm{C}_{13} \mathrm{H}_{16} \mathrm{~N}_{8} \mathrm{O}_{4}$ $\mathrm{m} / \mathrm{z} 307(\mathrm{M}+\mathrm{H}){ }^{+}$

1-[[1-[(2-Hydroxyethoxy)methyl]-1,2,3-triazol-4-yl]methyl](3-chloro-6-nitro indazole) 13d. Solid, mp $89-92{ }^{\circ} \mathrm{C},{ }^{1}{ }^{1} \mathrm{H}$ NMR, DMSO-d 6 , $\delta 3.50\left(\mathrm{~m}, 4 \mathrm{H}, \mathrm{CH}_{2} \mathrm{CH}_{2}\right), 4.71(\mathrm{~s}, 1 \mathrm{H}, \mathrm{OH}), 5.69$ (s, $\left.2 \mathrm{H}, \mathrm{T}-\mathrm{CH}_{2}-\mathrm{B}\right), 5.93\left(\mathrm{~s}, 2 \mathrm{H}, \mathrm{OCH}_{2} \mathrm{~N}\right) ; 7.22\left(\mathrm{~s}, 2 \mathrm{H}, \mathrm{NH}_{2}\right), 7.94 ; 8.05(\mathrm{~m}, 2 \mathrm{H}, \mathrm{H}-5, \mathrm{H}-4) ; 8.33$ (s, $1 \mathrm{H}, \mathrm{H}-5$ (triazole)), 8.99(d, $1 \mathrm{H}, \mathrm{H}-7) .{ }^{13} \mathrm{C}$ NMR, DMSO-d 6 : 45.05; 60.44; 71.58; 78.85; 108.70; 116.84; (2xC) 121.19; (2xC) 124.35; 140.05; 142.97; 147.75. ESI-MS, $\mathrm{C}_{13} \mathrm{H}_{13} \mathrm{~N}_{2} \mathrm{O}_{4} \mathrm{Cl}$ $\mathrm{m} / \mathrm{z}=352.9$

1-[[1-[(2-Hydroxyethoxy)methyl]-1,2,3-triazol-4-yl]methyl](3-bromo-6-nitroindazole) 13c. Solid, mp $116-119{ }^{\circ} \mathrm{C},{ }^{1} \mathrm{H}$ NMR, DMSO-d 6 , $\delta 3.50$ ( m, 4H, $\mathrm{CH}_{2} \mathrm{CH}_{2}$ ); 4.71 ( sl, 1H, OH); 5.69 (s, 2H, T-CH $\left.{ }_{2}-\mathrm{B}\right), 5.93\left(\mathrm{~s}, 2 \mathrm{H}, \mathrm{OCH}_{2} \mathrm{~N}\right) ; 7.22$ (s, $\left.2 \mathrm{H}, \mathrm{NH}_{2}\right) ; 8.05 ; 8.24$ ( m, 2H, H-5, H-4); 8.54 (s, 1H, H-5 (triazole)); 9.19 (d,1H, H-7). ${ }^{13} \mathrm{C}$ NMR, DMSO-d 6 : 45.74; 60.93; 72.20; 79.49; 109.19; 117.49; (2xC) 122.40; (2xC) 125.53; 140.53; 143.64; 148.36. ESI-MS, $\mathrm{C}_{13} \mathrm{H}_{13} \mathrm{~N}_{2} \mathrm{O}_{4} \mathrm{Br}$ $\mathrm{m} / \mathrm{z}=396.8$.

1,3-Bis-[[1-[(2-hydroxyethoxy)methyl]-1,2,3-triazol-4-yl]methyl]uracil 15a. Viscous residue, ${ }^{1} \mathrm{H}$ NMR, DMSO-d 6 , $\delta 3.45\left(\mathrm{~m}, 8 \mathrm{H}, 2 \mathrm{x}\left(\mathrm{CH}_{2} \mathrm{CH}_{2}\right)\right) ; 4,72(\mathrm{~s}, 2 \mathrm{H}, 2 \mathrm{x}(\mathrm{OH})) ; 5.05(\mathrm{~s}, 4 \mathrm{H}, 2 \mathrm{x}(\mathrm{T}-$ $\left.\mathrm{CH}_{2}-\mathrm{B}\right)$ ); 5.71 (s, 4H, 2x(OCH$\left.\left.-\mathrm{O}\right)\right) ; 5.79(\mathrm{~d}, 1 \mathrm{H}, \mathrm{H}-5, J=7.89 \mathrm{~Hz}) ; 7.86(\mathrm{~d}, 1 \mathrm{H}, \mathrm{H} 6, J=7.86 \mathrm{~Hz})$; 8.08 (s, $1 \mathrm{H}, \mathrm{H}-5$ (triazole $)$ ); 8.25 (s, $1 \mathrm{H}, \mathrm{H}-5$ (triazole) $).{ }^{13} \mathrm{C}$ NMR, DMSO-d 6 : 44.12; 46.33; $60.33 ; 63.35 ; 71.52 ; 71.59 ; 78.69 ; 78.84 ; 101.12 ; 124.38 ; 124.73 ; 143.12 ; 143.89 ; 144.89$; 151.33; 162.60. ESI-MS, $\mathrm{C}_{16} \mathrm{H}_{2} \mathrm{~N}_{8} \mathrm{O}_{6} \mathrm{~m} / \mathrm{z}=422.9$

1,3-Bis-[[1-[(2-hydroxyethoxy)methyl]-1,2,3-triazol-4-yl]methyl]thymine 15b. Solid, mp 72 $76^{\circ} \mathrm{C} ;{ }^{1} \mathrm{H}$ NMR, DMSO-d 6 ; $\delta 1.83\left(\mathrm{~s}, 3 \mathrm{H}, \mathrm{CH}_{3}\right) ; 3.48\left(\mathrm{~m}, 8 \mathrm{H}, 2 \mathrm{x}\left(\mathrm{CH}_{2} \mathrm{CH}_{2}\right)\right) ; 4.74(\mathrm{~s}, 2 \mathrm{H}$, $2 \mathrm{x}(\mathrm{OH})) ; 5.04$ (s, 4H, 2x(T-CH $-\mathrm{B})) ; 5.19$ (s, 4H, 2x( $\left.\left(\mathrm{OCH}_{2}-\mathrm{T}\right)\right) ; 7.25$ (d, 1H, H-6); 8,07 (s, 1H, $\mathrm{H}-5$ (triazole)), 8.25 (s, $1 \mathrm{H}, \mathrm{H}-5$ (triazole)). ${ }^{13} \mathrm{C}$ NMR, DMSO-d 6 : $12.53 ; 43.35 ; 45.75 ; 59.72$; $62.73 ; 70.92 ; 70.99 ; 78.07 ; 78.23 ; 108.15 ; 123.79 ; 124.09 ; 140.10 ; 142.66 ; 143.06 ; 150.57$; 162.73. ESI-MS, $\mathrm{C}_{17} \mathrm{H}_{24} \mathrm{~N}_{8} \mathrm{O}_{6} \mathrm{~m} / \mathrm{z}=436.9$

\section{References}

1. Chen, M. D.; Lu, S. J.; Yuag, G. P.; Yang, S. Y.; Du, X. L. Heterocycl. Commun. 2000, 6, 421.

2. Diana, G. D., Nitz, J. J. EP 566 199, 1993. 
3. Manfredini, S.; Vicentini, C. B.; Manfrini, M.; Bianchi, N.; Rutigliano, C.;Mischiati, C.; Gambari, R. Bioorg. Med. Chem. 2000, 8, 2343.

4. Sidwell, R. W.; Huffman, J. H.; Khare, G. P.; Allen, L. B.; Witkowski, J. T.; Robins, R. K. Science 1972, 177, 705.

5. (a) De Clercq, E. Clin. Microbiol. Reviews 1997, 10, 674. (b) De Clercq, E. Nat. Rev. Drug Discovery 2002, 1, 13. (c) Alvarez, R.; Velazquez, S.; San-Felix, A.; Aquaro, S.; De Clercq, E.; Perno, C.-F.;Karlsson, A.; Balzarini, J.; Camarasa, M. J. J. Med. Chem. 1994, 37, 4185.

6. Whiting, M.; Tripp, J. C.; Lin, Y. C.; Lindstrom, W.; Olson, A. J.; Elder, J. H.; Sharpless, K. B.; Fokin, V. V. J. Med. Chem. 2006, 49, 7697.

7. Lazrek, H. B.; Taourirte, M.; Oulih, T.; Barascut, J. L.; Imbach, J. L.; Pannecouque, C.; Witrouw, M.; De Clercq, E. Nucleosides Nucleotides Nucleic acids 2001, 20, 1949.

8. (a) Kolb, H. C.; Sharpless, K. B. Drug Discov. Today 2003, 8, 1128. (b) Bock, V. D.; Hiemstra, H.; van Maarseveen, J. H. Eur. J. Org. Chem. 2006, 51.

9. (a) Huisgen, R. Angew. Chem., Int. Ed. 1963, 2, 565. (b) 1,3-Dipolar Cycloaddition Chemistry; Padwa, A., Ed.; Wiley: New York, 1984; pp 1-176. (c) Comprehensive Organic Synthesis; Trost, B. M., Ed.; Pergamon: Oxford, 1991; Vol. 4, pp 1069-1109.

10. (a) Moorhouse, A. D.; Santos, A. M.; Gunaratnam, M.;Moore, M.; Neidle, S.; Moses, J. E. J. Am. Chem. Soc. 2006, 128, 15972. (b) Lee, L. V.; Mitchell, M. L.; Huang, S.-J.; Fokin, V. V.; Sharpless, K. B.; Wong, C.-H. J. Am. Chem. Soc. 2003, 125, 9588.

11. (a) Wu, P.; Feldman, A. K.; Nugent, A. K.; Hawker, C. J.;Scheel, A.; Voit, B.; Pyun, J.; Frechet, J. M. J.; Sharpless,K. B.; Fokin, V. V. Angew. Chem., Int. Ed. 2004, 43, 3928. (b) Wu, P.; Malkoch, M.; Hunt, J. N.; Vestberg, R.;Kaltgrad, E.; Finn, M. G.; Fokin, V. V.; Sharpless, K. B.;Hawker, C. J. Chem. Commun. 2005, 5775. (c) Rozkiewicz, D. I.; Janczewski, D.; Verboom, W.; Ravoo,B. J.; Reinhoudt, D. N. Angew. Chem., Int. Ed. 2006, 45, 5292 .

12. (a)Wang, Q.; Chan, T. R.; Hilgraf, R.; Fokin, V. V.; Sharpless, K. B.; Finn, M. G. J. Am. Chem. Soc. 2003, 125, 3192. 10. (b) Speers, A. E.; Adam, G. C.; Cravatt, B. F. J. Am. Chem. Soc. 2003, 125, 4686. (c) Speers, A. E.; Cravatt, B. F. Chem. Biol. 2004, 11, 535. (d) Burley, G. A.; Gierlich, J.; Mofid, M. R.; Nir, H.; Tal, S.; Eichen, Y.; Carell, T. J. Am. Chem. Soc. 2006, $128,1398$.

13. Chittepu, P.; Sirivolu, V. R.; Seela, F. Bioorg. Med. Chem. 2008, 16, 8427.

14. Yan, Z. Y.; Zhao, Y. B.; Fan, M. J.; Liu, W. M.; M.Liang, Y. Tetrahedron 2005, 61, 9331.

15. El Akri, K.; Bougrin, K.; Balzarini, B. J.; Faraj, A.; Benhida, R. Bioorg. Med. Chem Lett. 2007, 17, 6656. 